\title{
Mitochondrial Dysfunction in a Patient with 8q21.11 Deletion and Charcot-Marie-Tooth Disease Type 2K due to GDAP1 Haploinsufficiency
}

\author{
Dmitriy Niyazov Diane Africk \\ Department of Pediatrics, Ochsner Clinic Foundation, New Orleans, La., USA
}

\author{
Key Words \\ Charcot-Marie-Tooth disease type $2 \cdot 8 q 21.11$ deletion . \\ GDAP1 - Mitochondrial dysfunction
}

\begin{abstract}
Unbalanced chromosomal rearrangements typically cause multiple organ system involvement including neurodevelopmental deficits. It is atypical, however, to experience developmental and neurological regression. We describe a female with intellectual disability, failure to thrive, short stature, multiple congenital anomalies, and dysmorphic features and a previously diagnosed de novo 8 q21.11 deletion at the age of 7 . However, at the age of 11 , she experienced neurological and developmental regression. The GDAP1 gene encoding ganglioside-induced differentiation-associated protein 1 was deleted in the patient as a part of the contiguous gene syndrome. We argue that haploinsufficiency of GDAP1 could have contributed to the proband's regression based on its involvement in mitochondrial function and a signal transduction pathway in neuronal development.
\end{abstract}

(c) 2015 S. Karger AG, Basel

Multiple organ system anomalies, including structural and developmental, usually prompt investigation for chromosomal imbalances due to advances in array-based comparative genomic hybridization (aCGH) [Watson et al., 2014]. Microdeletions or microduplications can in- volve multiple genes and affect many organs as a result of the contiguous gene syndrome. If one of the genes as a part of a chromosomal rearrangement encodes mitochondrial function, the patient can have mitochondrial dysfunction in addition to multiple congenital anomalies, dysmorphisms and developmental delay. While the latter is a common feature of chromosomal disorders, neurodevelopmental regression is atypical. Mitochondrial disease is well known to be extremely heterogeneous and is associated with regression [Chinnery et al., 2000]. We present a female with an $8 \mathrm{q} 21.11$ deletion causing a contiguous gene syndrome including the GDAP1 gene (OMIM 606598; http://omim.org/entry/606598) implicated in mitochondrial dysfunction and Charcot-MarieTooth disease type $2 \mathrm{~K}$ (CMT2K).

\section{Case Report}

We saw the female proband for the first time at the age of 12 , and she had a history of developmental delay, intellectual disability, failure to thrive, short stature, cleft palate, congenital heart disease, and dysmorphic features. At the age of 7, she was found to have a de novo 11.8-Mb deletion in the 8q21.11 chromosomal region, which explained most of her symptoms until 11 years of age when she experienced a regression in language and worsening gait and fatigue which required intermittent use of a wheelchair. Her electromyography showed a mild axonal neuropathy. Her brain MRI showed a global cerebral atrophy which was slightly more pronounced than 5 years prior. Additional novel symptoms included tremor and hoarseness of voice.

\section{KARGER 125}

(c) 2015 S. Karger AG, Base

$1661-8769 / 15 / 0064-0204 \$ 39.50 / 0$

E-Mail karger@karger.com

www.karger.com/msy
Dmitriy Niyazov, MD

Department of Pediatrics, Ochsner Clinic Foundation 1315 Jefferson Hwy

New Orleans, LA 70121 (USA)

E-Mail dniyazov@ochsner.org 
Fig. 1. The 11.8-Mb deletion detected by the $105 \mathrm{~K}$ oligonucleotide aCGH in $8 \mathrm{q} 21.118 \mathrm{q} 21.2$.

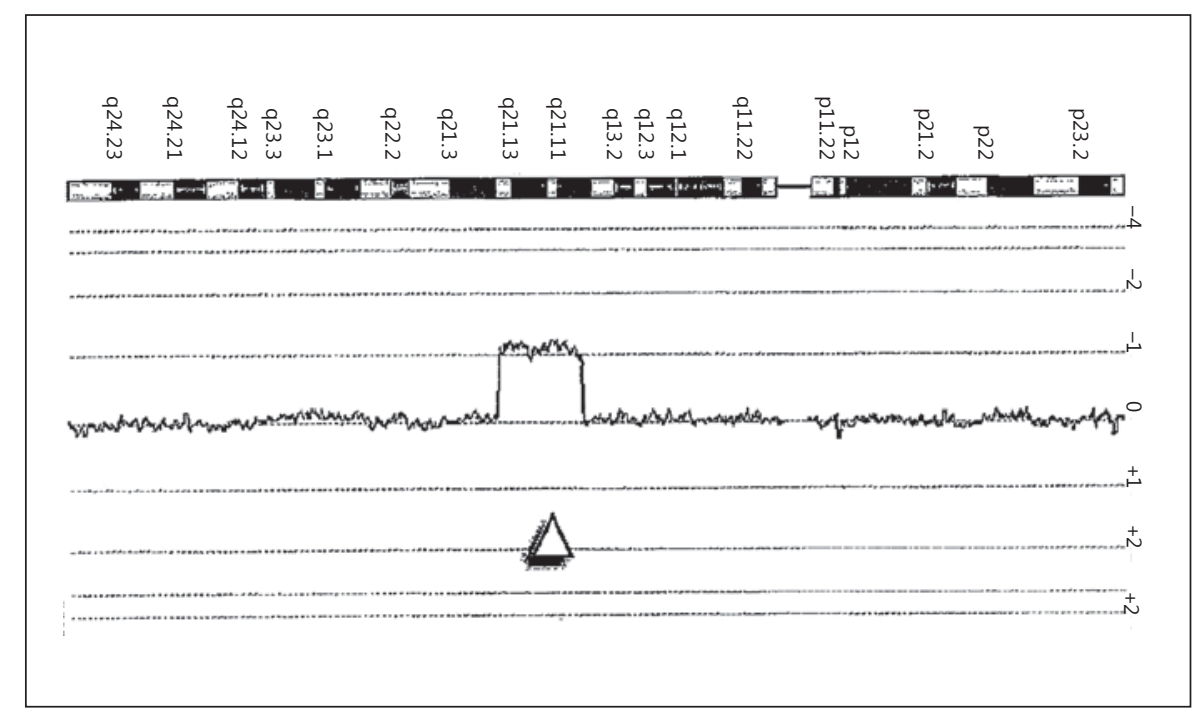

\section{Methods}

Previous aCGH analysis was performed using 105K oligonucleotide array (Agilent), and the deletion was confirmed in the proband and ruled out in both parents by FISH. The microdeletion analysis for contiguous genes was performed using the Genome Browser (http://genome.ucsc.edu). Sanger sequencing and exon array of the GDAP1 gene were performed to rule out point mutations and microrearrangements on the opposite (non-deleted) gene. Mitochondrial evaluation was performed by obtaining metabolic studies in blood and urine (lactate and lactate/pyruvate ratio, carnitine, alanine, etc.). The proband's skeletal muscle was analyzed by immunohistochemistry, immunofluorescence, mitochondrial DNA, and electron transport chain.

\section{Results}

The previous $105 \mathrm{~K}$ oligonucleotide aCGH detected a de novo 11.8-Mb deletion (fig. 1) in 8q21.118q21.2 $(74,915,888-86,743,493)$, and FISH confirmed the deletion in the proband and excluded it in both parents. The Genome Browser showed more than 35 genes in the deleted region, but the GDAP1 encoding ganglioside-induced differentiation-associated protein 1 was considered to be the most likely candidate explaining the patient's clinical phenotype. Sanger sequencing was negative for point mutations and exon array of the GDAP1 gene only showed one deletion and no microrearrangements on the opposite (non-deleted) gene. The girl's multiple metabolic studies in blood and urine were suggestive of mitochondrial dysfunction, including high lactate and lactate/pyruvate ratio, low plasma and urine carnitine,
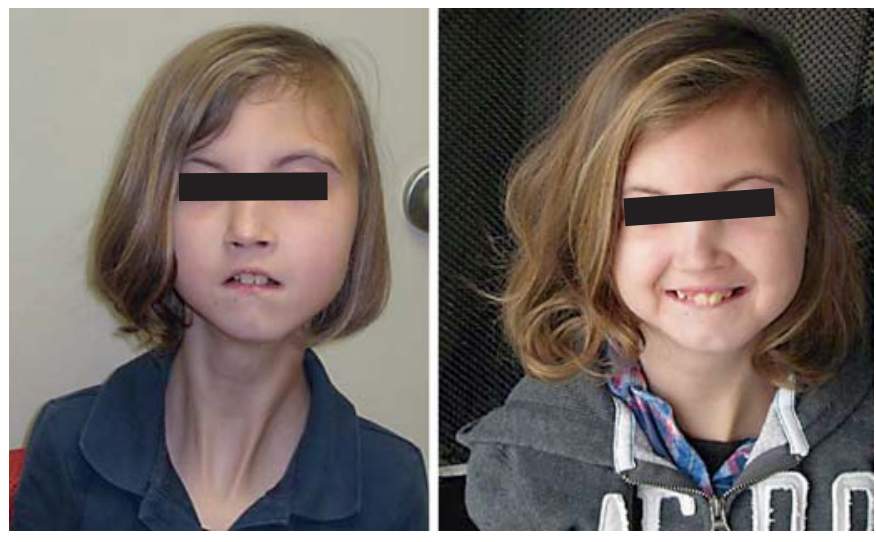

Fig. 2. The proband before and 3 months after gastrostomy placement and mitochondrial vitamin cocktail.

high alanine and glutamine, etc. The proband's muscle biopsy (performed with a gastrostomy tube placement) revealed a widespread reduced expression in the mitochondrial complex I on immunofluorescent studies and mildly decreased activity of I+III complex on electron transport chain analysis but insufficient to satisfy a minor Walker criterion. Treatment of her mitochondrial dysfunction included improved nutrition via gastrostomy (fig. 2) and a vitamin cocktail including CoQ10, creatine and lipoic acid. The girl experienced a stabilization of her neurological status and an improvement in several developmental parameters including motor and language skills. 


\section{Discussion}

To our knowledge, the girl's complex and unique presentation, course of the disease and treatment have not been previously published. The GDAP1 gene deletion (as a part of the large 8q21.11 deletion) could have contributed to her regression, since it encodes the GDAP1 protein (ganglioside-induced differentiation-associated protein 1). The latter has been shown to be involved in mitochondrial function and a signal transduction pathway in neuronal development [Knott et al., 2008; Cassereaua et al., 2011; Palmomeres et al., 2011; Zimoń et al., 2011]. Mutations in this gene have been associated with both autosomal recessive and dominant CMT2K, which is a progressive disorder that affects the peripheral nerves [Bird, 1998]. The autosomal dominant form is less severe and presents later in life, which is more consistent with the proband's history (negative GDAP1 sequencing and exon array only showing one deletion made an autosomal recessive form unlikely).

The proband's autosomal dominant CMT2K likely caused a mild axonal neuropathy with slow progression and ataxic gait, which is the most common initial symptom. Disease progression is slow, and patients typically remain ambulatory which was comforting for the parents to know. As a matter of fact, her strength, if anything, improved with treatment (nutrition with the help of gastrostomy and vitamin cocktail), and she hardly uses a wheelchair anymore. Weakness and atrophy in CMT2K is mainly restricted to distal muscles of the lower and upper limbs, but some patients develop a mild proximal weakness. Unilateral or bilateral vocal fold paresis can be seen, and the girl was referred to laryngoscopy and indeed found to have a right vocal fold paresis, which is current- ly monitored by her otolaryngologist to prevent catastrophic complications.

The proband's multiple metabolic studies in blood and urine were suggestive of mitochondrial dysfunction (high lactate and lactate to pyruvate ratio, low carnitine, high glutamine, etc.). Moreover, her muscle biopsy revealed a decreased activity of I+III complex on electron transport chain and widespread reduced expression of complex I on immunofluorescent studies. Even though the decrease in her I+III complex activity was not enough to satisfy a minor Walker criterion, it is still significant as it shows potential secondary mitochondrial dysfunction.

In conclusion, the patient likely had a mitochondrial dysfunction due to the deletion and haploinsufficiency of the GDAP1 gene which resulted in her neurodevelopmental regression. Her symptoms significantly improved after proper nutrition with the help of gastrostomy and vitamin cocktail. Her motor prognosis appears to be good based on the autosomal dominant rather than the recessive CMT2K. Finally, her vocal cord paresis is carefully monitored to prevent complications such as airway obstruction.

\section{Statement of Ethics}

All procedures performed were in accordance with the ethical standards of the institutional and/or national research committee. Informed consent was obtained from the parents according to our institutional protocol.

\section{Disclosure Statement}

The authors have no conflicts of interest to declare.

\section{References}

Bird TD: Charcot-Marie-Tooth neuropathy type Chinnery PF: Mitochondrial disorders overview, 2, in Pagon RA, Adam MP, Bird TD, et al (eds): GeneReviews ${ }^{\circledR}$ [Internet]. (University of Washington, Seattle 1998). http://www. ncbi.nlm.nih.gov/books/NBK1285.

Cassereau J, Chevrollier A, Gueguen N, Desquiret V, Verny C, et al: Mitochondrial dysfunction and pathophysiology of Charcot-MarieTooth disease involving GDAP1 mutations. Exp Neurol 227:31-41 (2011). in Pagon RA, Adam MP, Ardinger HH, et al (eds): GeneReviews ${ }^{\circledR}$ [Internet]. (University of Washington, Seattle 2000). http://www. ncbi.nlm.nih.gov/books/NBK1224.

Knott A, Perkins G, Schwarzenbacher R, BossyWetzel E: Mitochondrial fragmentation in neurodegeneration. Nat Rev Neurosci 9:505518 (2008).

-Palomares M, Delicado A, Mansilla E, de Torres ML, Vallespín E, et al: Characterization of a 8q21.11 microdeletion syndrome associated with intellectual disability and a recognizable phenotype. Am J Hum Genet 89:295-301 (2011).
C, Marques-Bonet T, Sharp A, Mefford $\mathrm{H}$ : The genetics of microdeletion and microduplication syndromes: an update. Annu Rev Genom Hum Genet 15:215-244 (2014).

Zimoń M, Baets J, Fabrizi GM, Jaakkola E, Kabzińska D, et al: Dominant GDAP1 mutations cause predominantly mild CMT phenotypes. Neurology 77:540-548 (2011).

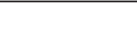

